\title{
Analysis of hydrogenated zirconium alloys irradiated with gamma - rays
}

\author{
Askar Askhatov ${ }^{1, *}$, Vitaliy Larionov ${ }^{1}$, and Victor Kudiyarov ${ }^{1}$ \\ ${ }^{1}$ Tomsk Polytechnic University, 634050 Tomsk, Russia
}

\begin{abstract}
The paper represents the investigations concerning the geometrical size effect of hydrogenated zirconium alloys $(\mathrm{Zr}-1 \mathrm{Ni}-\mathrm{H})$ during gamma-ray irradiation on the amount of energy absorbed. The results have shown that the less the cross-sectional dimensions of the sample or product is, the less energy is absorbed. The paper provides theoretical calculations. The zirconium sample with a cross-section of $2.8 \times 2.8 \mathrm{~cm}$ absorbs $30-35 \%$ of the energy of the incident gamma-ray flow. The increase in the cross-section of a product up to $28 \mathrm{~cm}$ leads to the increase in the absorbed energy by more than 2 times. At the same time, the thickness of the product is constant. This effect is explained by the multiple scattering of gamma-rays. It leads to the nonuniform distribution of defects which can accumulate hydrogen and should be considered when developing the analysis methods. These edge effects are confirmed by the measurement of the thermal electromotive force for the samples of zirconium alloys before hydrogenation and gamma-ray irradiation, and after irradiation.
\end{abstract}

\section{Introduction}

At present, the different modifications of known metal are used to obtain new materials for the aerospace industry. The development of technologies and equipment requires the information on the mechanisms and characteristics of physical and chemical processes, the characteristics of the elemental composition and the structural phases of products [1-5]. Most of the products are exploited under ionizing radiation of different nature: electrons, ions, gamma-rays $[1,2,5-9]$. Radiation is used in the continuous and pulsed modes [2, 6, 7]. It is of particular interest to study the effect of radiation on hydrogenated materials [2, 5]. The properties of these materials are of particular interest to the aerospace materials science. In recent years, these problems have become urgent to researchers in different scientific areas [9], since there is a possibility to achieve the deep and controlled restructuring of metals and alloys on the different structure levels [9]. Various physical methods are used for the analysis of these materials [1-3]. The goal of this work is to study the gamma-ray energy accumulated by the zirconium samples of different sizes. The amount of absorbed energy depends both on the material properties and geometrical sizes. To explain this fact, an analytical calculation method is used [10]. The direct analysis of

\footnotetext{
* Corresponding authors: askhatov10@mail.ru
} 
changes in the properties of irradiated materials was conducted by the thermal electromotive force method [11].

\section{Materials and methods}

The samples of the $\mathrm{Zr} 1 \% \mathrm{Ni}$ alloy were in the form of a $20 \times 20 \mathrm{~mm}$ rectangle with a thickness of $1 \mathrm{~mm}$ and disc-shaped with a radius of $2 \mathrm{~cm}$ and a thickness of $2 \mathrm{~mm}$. The sample hydrogenation was conducted using an installation PCI_ "Gas Reaction Controller» by the Sieverts method at the temperatures $\mathrm{T}=450-600^{\circ} \mathrm{C}$ and the pressure of $0.66 \mathrm{~atm}$. The hydrogen concentration was varied from $\mathrm{C}=690$ to $\mathrm{C}=5000 \mathrm{ppm}$. The absolute hydrogen concentration in the alloy was determined using a RHEN 602 analyzer (LECO). Irradiation with the $\mathrm{Co}^{60}$ gamma-rays was conducted using an installation IRT -2000 (TPU) with different doses of gamma-rays up to $2,6.10^{18} \gamma$-sq $/ \mathrm{cm}^{2}$. The thermal electromotive force was measured using a T-3SP device and a V7-78/1 voltmeter. The thermal probe was made of a gold rod with a diameter of $1 \mathrm{~mm}$.

\section{Results}

The absorption of gamma-rays with an energy of $80 \mathrm{keV}$ was anylized using the Goryachev-Larionov model [10]. The calculations were performed for the following initial data: the geometric sizes of the target sample $\left(\mathrm{y}_{0}, \mathrm{z}_{0}, x_{o}=d\right)$, the attenuation coefficient $\mu(E)$, the total cross section of absorption for Compton scattering $\Sigma_{\mathrm{g}}^{\mathrm{h}}$ and photoeffect $\Sigma_{\mathrm{g}}^{\mathrm{f}}$, the total cross section of scattering $\Sigma_{\mathrm{s}}$, the integral «effective» probability of X-ray photon survival for Compton scattering $\Lambda_{k}=\Sigma_{s} /\left(\Sigma_{s}+\Sigma_{g}^{k}\right)$, the integral «effective» probability of photon survival during the process of photoeffect $\Lambda_{\Phi}=\Sigma_{s} /\left(\Sigma_{s}+\Sigma_{g}\right)$, the integral probability of photon absorption $P=1-\Lambda_{\Phi} \Lambda_{k}$ ), and the initial intensity for the incident flow of gamma-ray emission is equal to 1 . The amount of energy absorbed in terms of the initial flow is given by

$$
E_{p}=g \frac{\left[1-A_{\infty}\left(y_{0}, z_{0}, E_{0}\right)\right]\left\{1-\exp \left[-K\left(y_{0}, z_{0}, E_{0}\right) \mu d\right]\right\}}{1+A_{\infty}\left(y_{0}, z_{0}, E_{0}\right) \exp \left[-K\left(y_{0}, z_{0}, E_{0}\right) \mu d\right]}
$$

The example of calculation by the formula (1) of $\gamma$ - ray absorption in the samples of different geometric sizes (zirconium) is illustrated in Figure 1.

The maximum absorption of gamma-rays for this energy is observed for the samples with a cross-section of $0.556 \times 0.556 \mathrm{~m}$ and more. But, for example, for a target with a crosssection of $2.8 \times 2.8 \mathrm{~cm}$, relative absorption is $\sim 40 \%$. The dependence of $\gamma$-rays absorbed on the thickness of the target $(x)$ is obvious and increases with increasing $x$ for the samples of any cross-section. The analysis of calculations also shows that the energy is absorbed nonuniformly over the sample area due to intensive leaving $\gamma$-rays through the lateral surface of the target sample.

It can be supposed that the defects will be accumulated nonunifromly according to this effect. Therefore, for analyzing the sample by various methods, there is a need to consider this peculiarity when a sensor moves on the surface of the sample irradiated. It should be noted that this peculiarity of the energy absorption during the multiple scattering of gammarays should be considered for the small size samples exposed to irradiation and used in the preliminary experiments. In the calculations, the mass attenuation coefficient was taken to be $1.64\left(\mu / \rho, \mathrm{cm}^{2} / \mathrm{g}\right)$ for zirconium. 


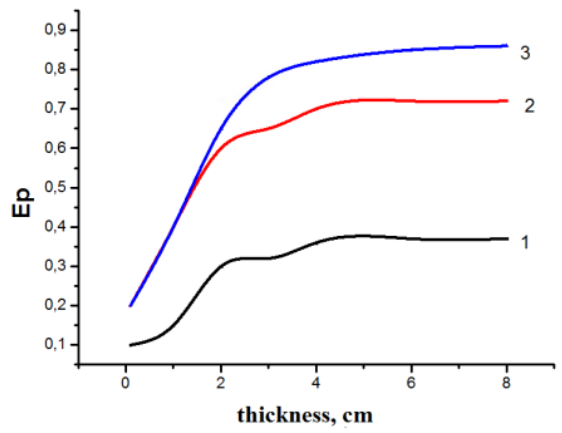

Fig. 1. Absorbed energy (in fractions of the incident flow) versus the sample thickness. (1) the size of cross-section is $2.8 \times 2.8 \mathrm{~cm}$; (2) $-0.28 \times 0.28 \mathrm{~m}$; (3) - $0.33 \times 0.33 \mathrm{~m}$.

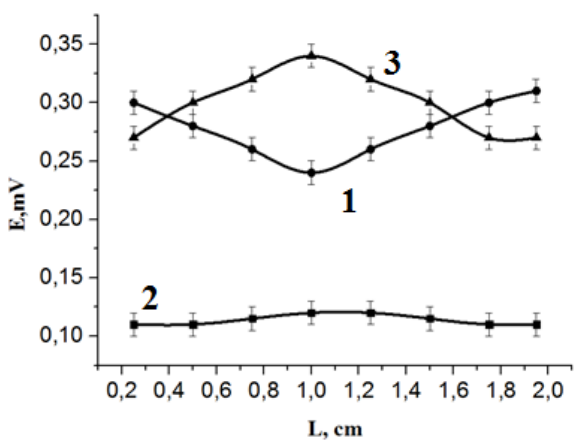

Fig. 2. Thermal electromotive force versus the sensor position on the sample surface. (1) is the hydrogenated sample before gamma-ray irradiation, (2) is the initial commercial $\mathrm{Zr}-1 \mathrm{Ni}$ alloy before hydrogenation, (3) is the irradiated and hydrogenated sample $\left(\mathrm{C}_{\mathrm{H}}=3000 \mathrm{ppm}\right)$.

The value of the thermal electromotive force for the initial sample is constant within the measurement error (Figure 2). This value is increased by more than 3 times, which substantially exceeds the measurement error. Corrosion can occur on the surface of hydrogenated zirconium. It is manifested in the form of a strip. A probe for measuring the thermal electromotive force along and perpendicular to the defect registers the jump of the thermal electromotive force (15-20\%) depending on the temperature.

\section{Discussion}

The highest change of the thermal electromotive force versus the coordinates of the sample was found for the irradiated sample. Edge effects in the hydrogenated sample were caused by the heterogeneity of hydrogenation from the gaseous atmosphere, using the Sieverts method. It should be noted, that this property should be considered in the analysis of physical quantities versus the hydrogen concentration. The integral method for determining the concentration of hydrogen in metals (LECO method) does not consider this peculiarity. The dependence of the thermal electromotive force on the coordinates of the probe for the irradiated sample is reversed with respect to the unirradiated sample. This effect can not be explained by the heterogeneity of hydrogenation. Therefore, the most acceptable explanation is the influence of the edge effect. The edge effect is caused by the intensive leaving gamma-rays through the lateral surface of the sample. This is confirmed by the 
qualitative calculation shown in Figure 1. Irradiation accelerates the loss of hydrogen by the test material.

This behavior of irradiated materials can be connected with the creation of additional hydrogen vacancies during gamma-ray irradiation. In the irradiated samples, this process is caused mainly by hydrogen diffusion in internal flows which can represent the grain boundaries. In addition, the irradiation of the alloy leads to the change in the concentration of charge carriers, which results in the different behavior of irradiated and unirradiated materials. The measurements conducted in the mutually perpendicular directions along the sample can show the change in the thermal electromotive force up to $15 \%$. This jump in the value of the thermal electromotive force can be explained by the change in the chemical composition, as well as by the deformation of the alloy surface.

\section{Conclusions}

The theoretical and experimental results have shown that the geometrical sizes of the hydrogenated zirconium alloys $\mathrm{Zr}-1 \mathrm{Ni}-\mathrm{H}$ influence on the amount of energy absorbed during gamma-ray irradiation. The calculations demonstrated that the smaller the lateral sizes of the sample or the product were, the less energy was absorbed, regardless the thickness of the sample. The effect is explained by the multiple scattering of gamma-rays. It leads to the nonuniform distribution of defects, which can accumulate hydrogen and should be considered when developing the analysis methods. The presence of edge effects was experimentally confirmed by the measurement of the thermal electromotive force for the zirconium alloy samples before hydrogenation and gamma-ray irradiation and after irradiation. The factor of heterogeneous hydrogenation of samples by the Sieverts method was considered in the measurements of edge effects. The value of thermal electromotive force is changed by more than 1.6 times and depends on the amount of hydrogen injected into the target, the target size and the coordinates of the probe position on the target body.

\section{References}

1. K. Oura, V.G. Lifshits, A.A. Saranin, Vvedenie v fiziku poverkhnosti (Nauka, Moscow, 2006) (in Russian)

2. I.P. Chernov, S.V. Ivanova, M.Kh Krening, N. Koval', V.V. Larionov, A.M. Lider, N.S. Pushilina, E.N. Stepanova, O.M. Stepanova, Yu.P. Cherdantsev, Technical Physics 57, 392 (2012)

3. V.V. Ovchinnikov, UFN 178, 9 (2008)

4. E.A. Denisov, T.N. Kompaniets, ZhTF 71, 2 (2001)

5. V.V. Larionov, N.N. Nikitenkov, Yu. I. Tyurin, Technical Physics 61, 5 (2016)

6. I.P. Chernov, A.S. Rusetsky, D.N. Krasnov,V.V. Larionov, T.I. Sigfusson, Yu.I. Tyurin, Journal of Engineering Thermophysics 20, 4 (2011)

7. Yu.S. Nechaev, UFN 178, 7 (2008)

8. J. Als-Nielsen, D. McMorrow, Elements of Modern X-Ray Physics (Wiley, New York, 2001)

9. A.V. Gapontsev, V.V. Kondratyev, UFN 173, 10 (2003)

10. B.V. Goriachev, V.V. Larionov, S.B. Mogilnitskii, B.A. Saveliev, Atomic energy 62, 5 (1987) (in Russian)

11. A.M. Lider, V.V. Larionov, M. Kroening, V.N. Kudiiarov IOP Conf. Ser.: Mater. Scien. and Engin 132, 1 (2016) 